\title{
Sistema Integrado de Gestão (SIG): Análise da percepção dos docentes e gestores dos cursos do Centro de Ciências Sociais Aplicadas da Universidade Federal de Sergipe
}

Este estudo teve como objetivo analisar o Sistema Integrado de Gestão (SIG) da Universidade Federal de Sergipe (UFS) no tocante a execução de tarefas e tomada de decisões, na percepção dos docentes e gestores dos cursos do Centro de Ciências Sociais Aplicadas (CCSA). Para tanto, a pesquisa foi fundamentada utilizando um embasamento teórico sobre sistemas de informações e Enterprise Resource Planning, mediante os quais buscou-se demonstrar que as organizações vêm identificando a necessidade de incorporar modernos sistemas de informações, dando ênfase a um tipo de abordagem mais sistêmica. Desse modo, a pesquisa foi caracterizada como descritiva com emprego do método quantitativo e qualitativo, junto aos entrevistados, docentes e gestores do CCSA, por meio da estratégia de estudo de caso. A partir dos resultados obtidos, foram evidenciados a caracterização do uso dos principais módulos do sistema, as principais facilidades de uso, as características das informações geradas pelo sistema e os problemas e possíveis soluções, que na percepção dos docentes e dos gestores, interferem diretamente na utilização e nos resultados obtidos através do sistema. Dessa forma, concluiu-se que o SIG atende a grande parte das demandas, porém, na percepção dos seus usuários, ainda necessita de melhorias em quesitos como capacitação, manutenção e flexibilidade.

\section{Integrated Management System (GIS): Analysis of the perception of teachers and managers of courses at the Center for Applied Social Sciences at the Federal University of Sergipe}

\begin{abstract}
This study aims to analyze the Integrated Management System (GIS) of the Federal University of Sergipe (UFS) regarding the execution of tasks and decision making, in the perception of teachers and managers of courses at the Center for Applied Social Sciences (CCSA). To this end, the research was based on a theoretical basis on information systems and Enterprise Resource Planning, through which it was sought to demonstrate that organizations have been identifying the need to incorporate modern information systems, emphasizing a type of more systemic approach. Thus, the research was characterized as descriptive using the quantitative and qualitative method, along with the interviewees, teachers and managers of the CCSA, through the case study strategy. From the results obtained, the characterization of the use of the main modules was evidenced. of the system, the main facilities of use, the characteristics of the information generated by the system and the problems and possible solutions, which in the perception of teachers and managers, directly interfere in the use and results obtained through the system. Thus, it was concluded that the GIS meets most of the demands, however, in the perception of its users, it still needs improvements in areas such as training, maintenance and flexibility.
\end{abstract}

Keywords: Information Systems; Enterprise Resource Planning; Integrated Educational Management System.

Topic: Sistemas e Tecnologia da Informação

Reviewed anonymously in the process of blind peer
Received: $\mathbf{2 0 / 0 3 / 2 0 2 0}$

Approved: 21/03/2020
Rafael Ribeiro de Lima (D)

Universidade Federal de Sergipe, Brasil http://lattes.cnpq.br/9182160507032907

http://orcid.org/0000-0002-1562-0961 rafakrd@hotmail.com

Maria Conceição Melo Silva Luft (ic Universidade Federal de Sergipe, Brasil http://lattes.cnpq.br/4838157048357924 http://orcid.org/0000-0003-2713-2700 ceicamelo.ufs@gmail.com

Carla Larisse Ferreira dos Santos (it) Universidade Federal de Sergipe, Brasil http://lattes.cnpq.br/2448601951180195 http://orcid.org/0000-0003-1271-1101 carlalarisse7@gmail.ocm
Referencing this:

LIMA, R. R.; LUFT, M. C. M. S.; SANTOS, C. L. F.. Sistema Integrado de Gestão (SIG): Análise da percepção dos docentes e gestores dos cursos do Centro de Ciências Sociais Aplicadas da Universidade Federal de Sergipe. Business Journal, v.2, n.1, p.39-53, 2020. DOI: http://doi.org/10.6008/CBPC2674-6433.2020.001.0004 


\section{INTRODUÇÃO}

A Tecnologia da Informação (TI) influencia de maneira direta os indivíduos e, por consequência, a sua performance no ambiente de trabalho, fazendo com que os procedimentos de sua responsabilidade se tornem cada vez mais efetivos e mantenham-se atualizados (ZANUZ, 2015). Desse modo, a TI impacta também no desempenho das organizações, ao afetar diretamente os resultados possíveis de serem alcançados, garantindo competitividade no mercado.

$\mathrm{Na}$ atualidade, é senso comum que os Sistemas de Informações (SIs) estão relacionados diretamente com os computadores, porém, a humanidade utiliza-se dos sistemas de informações desde os primórdios da civilização, com ou sem o uso da tecnologia. Através da análise de uma das definições do termo sistema, o porquê se torna evidente. O’Brien (2011) define sistema como sendo um conjunto de componentes interrelacionados com limites claramente definidos, que colaboram para a realização de objetivos em comum, produzindo resultados em um processo de transformação organizado. Dessa forma, um sistema pode ser definido como o agrupamento de pessoas e/ou recursos que possuem uma finalidade em comum.

Nessa perspectiva, ao associar a concepção de sistema com o significado do termo informação, “[...] dados apresentados em uma forma significativa e útil para os seres humanos" (LAUDON et al., 2012), obtémse uma noção básica do que são os sistemas de informações. Dentre os diversos tipos de Sls existentes, uma das classificações mais conhecidas e utilizadas é de Sistemas de Informações Gerenciais ou de Gestão (SIGs) que, de acordo com Kroenke (2017) "consistem no desenvolvimento e uso de sistemas de informação que ajudam as empresas a alcançar suas metas e objetivos". Isto é, os SIGs compreendem um conjunto organizado de pessoas, procedimentos, banco de dados e/ou dispositivos, que fornecem aos tomadores de decisão (gerentes ou gestores), as informações necessárias para se ter uma visão das operações regulares, de modo que possam controlar, organizar e planejar mais eficaz e eficientemente as atividades e os processos da instituição em que trabalham.

Esses sistemas têm influenciado as empresas como um todo, e com as instituições educacionais isso não poderia ser diferente. Os SIGs voltados para o setor educacional, também conhecidos como sistemas educacionais ou sistemas de gestão acadêmica, são plataformas, segundo Ziulkoski (2010), que visam atender às necessidades de gestão e planejamento de uma instituição de ensino, seja está pública ou privada, por meio de uma melhor utilização dos recursos físicos, humanos, materiais e financeiros. Cada vez mais os sistemas de gestão acadêmica vêm garantindo a essas instituições agregar qualidade as suas atividades e processos, tais como flexibilidade, eficiência, eficácia, rapidez e segurança.

Para Rocha Neto et al. (2009), as universidades modernas têm como característica importante bons sistemas de gestão acadêmica, o que lhes permite uma boa gestão da universidade como um todo, desde a parte administrativa, passando pela parte financeira e de recursos humanos, até questões educacionais como notas, acompanhamento de alunos e avaliações. Dessa maneira, através da utilização de informações de maneira mais eficiente e eficaz (o que antes da implementação desses sistemas não acontecia, principalmente devido à demora em capturar e processar os dados), os sistemas acadêmicos surgem quase 
que como uma obrigação para as instituições de ensino, com o objetivo de atender às necessidades e os desejos dos seus usuários (gestores, docentes, técnicos administrativos, discentes, entre outros), que são os principais interessados no desenvolvimento e aprimoramento desses sistemas.

Nesse contexto, o Sistema Integrado de Gestão (SIG) foi escolhido como objeto de estudo para esse artigo. Esse sistema é uma importante ferramenta que é utilizada de maneira muito abrangente, em especial para a comunidade acadêmica da Universidade Federal de Sergipe (UFS). Mediante a sua utilização, os atores mencionados têm acesso as suas diversas funcionalidades e podem realizar atividades que contribuem com a administração, com a excelência e com o aprimoramento dos cursos e da universidade como um todo, realizando processos que, por sua natureza, poderiam ser mais demorados e complexos.

Desse modo, encontrou-se uma oportunidade de expansão e aprofundamento do tema em uma unidade de ensino. Assim sendo, a questão de pesquisa na qual se baseou o estudo foi: Qual a percepção dos docentes e dos gestores dos cursos do Centro de Ciências Sociais Aplicadas (CCSA) da Universidade Federal de Sergipe (UFS) em relação ao Sistema Integrado de Gestão (SIG)?

Estudos sobre percepções de usuários acerca de sistemas de informações são realizados há anos, incluindo estudos sobre sistemas de gestão acadêmica (mais especificamente sobre o SIG). Através de pesquisas realizadas envolvendo o SIG nas bases Google Acadêmico e Periódico Capes, percebeu-se que na maior parte dos estudos o enfoque é dado em sua grande maioria aos discentes (graduação e pósgraduação), excluindo da análise os outros atores, usuários do SIG da comunidade acadêmica.

Nessa direção, o objetivo principal traçado foi analisar o Sistema Integrado de Gestão (SIG), e seus desmembramentos, no tocante a execução de tarefas e tomada de decisão, por meio da percepção dos gestores e dos docentes dos cursos do Centro de Ciências Sociais Aplicadas (CCSA) da Universidade Federal de Sergipe (UFS).

Para tanto, o artigo está estruturado em 5 seções. A primeira é esta introdução que apresenta o contexto geral do estudo. A segunda seção trata da fundamentação teórica acerca dos temas sistemas de informações e Enterprise Resource Planning. Na terceira seção, encontram-se os procedimentos metodológicos que nortearam a pesquisa. Na quarta seção, ocorre a apresentação do sistema escolhido para o estudo de caso, bem como a análise dos dados coletados apresentando os achados do estudo. Por fim, na quinta seção constam as considerações finais.

\section{REVISÃO TEÓRICA}

O conceito de sistema está ligado a um conjunto de elementos que interagem entre si para atingir um objetivo em comum (STAIR, 2001). De acordo com Stair et al. (2011), o SI é um conjunto de elementos inter-relacionados que coletam, manipulam e disseminam dados e informações, proporcionando mecanismo de retroação com o intuito de atingir um objetivo específico. Já Fernández et al. (2015) afirmam que os Sls são definidos pela cooperação de recursos materiais, humanos e informacionais organizados de uma maneira que permitam que as entradas (os dados) se transformem em saídas (informação). Desse modo, é correto 
afirmar que "um sistema de informação será, portanto, aquele que tem como núcleo central a informação e como finalidade a sua gestão" (FERNÁNDEZ et al., 2015).

Para atender tais objetivos, a utilização de recursos tecnológicos acaba sendo um aspecto muito importante, tendo em vista que, nos últimos anos, os equipamentos utilizados nas organizações têm evoluído cada vez mais rápido. Inclusive, para muitos autores, esses recursos tecnológicos são componentes essenciais de um SI, como é o caso de O'Brien (2011) e Laudon et al. (2012), que afirmam que os componentes de um SI podem ser compreendidos como pessoas, hardware, software, redes de comunicações e recursos de dados.

Todavia, os recursos tecnológicos servem apenas de auxílio para o bom funcionamento dos sistemas, e nem sempre são determinantes para o seu sucesso ou fracasso. Sendo assim, independentemente da plataforma em que eles funcionam, outros fatores influenciarão o resultado do processo. Um dos fatores que se deve ter uma atenção é o de integração de sistemas. Observa-se que, atualmente, os diversos autores que tratam deste tema têm reforçado a importância de se integrar informações e sistemas de informações dentro das organizações, pois muitas empresas acabam por ter diversos setores com sistemas diferentes e que não se conversam, impactando diretamente nos resultados dos processos, e consequentemente nos resultados das organizações em si.

Outro fator relevante é o da confiabilidade das informações. Muitas organizações pecam nesse quesito, pois acabam extraindo dados de forma não muito segura ou, quando o fazem de maneira correta, pecam na disseminação desta informação, principalmente através da interferência humana nesse processo, onde controladores e manipuladores dos sistemas erram ao inserir ou modificar dados no sistema, impactando de forma efetiva no resultado final dos procedimentos.

“Não se deve, contudo, privilegiar em excesso o aspecto tecnológico em detrimento das qualidades inerentes do SI em resolver problemas e controlar processos" (ANDRADE et al., 2001). De maneira geral e independentemente da plataforma que se utilize, de acordo com Gorla et al. (2010), sistemas que apresentam baixa qualidade resultam em baixa qualidade de informação. Sendo assim, deve-se observar que a qualidade do sistema é um fator importante no sucesso dos SIs que se utiliza, pois quando um sistema é de alta qualidade, ou seja, dentre outras características, apresenta capacidade de manutenção e flexibilidade, ele resulta em alta qualidade de informação, apresentando assim, conteúdos úteis e relevantes para o usuário (GORLA et al., 2010).

A sofisticação do sistema, quanto ao uso de tecnologias modernas e quanto a sua integração, contribui para a qualidade da informação por proporcionar a saída das informações em formato mais claro e consistente. Esta afirmação pode ser corroborada através do exemplo do ERP, que é basicamente um recurso de integração de dados e informações, que se utiliza de diversas ferramentas para suprir a necessidade de utilização dos outros tipos de sistema, além de integrar os diversos níveis e setores organizacionais existentes, facilitando assim, a gestão empresarial de uma maneira mais ampla e holística (JANNUZZI et al., 2014). 
Sistema Integrado de Gestão (SIG): Análise da percepção dos docentes e gestores dos cursos do Centro de Ciências Sociais Aplicadas da

Segundo Aparicio et al. (2018), logo quando esse tipo de sistema foi desenvolvido, apenas as grandes corporações o adotavam e o principal fator para essa restrição era o elevado custo para sua aquisição e manutenção, o que fazia as organizações despenderem muitos recursos. Com o advento da globalização e com a popularização dessa nova ferramenta, surgiram opções mais acessíveis dentro do mercado, o que fez com que empresas de médio porte também pudessem usufruir desse tipo de sistema (APARICIO et al., 2018). Laudon et al. (2012) afirmam que o ERP é basicamente uma evolução dos sistemas de informações, e que ele surge como solução para a necessidade de se ter um sistema único que integre todos os processos dos diversos níveis (operacional, tático e estratégico) e setores funcionais (produção, marketing, recursos humanos e finanças) de uma mesma organização. Turban et al. (2007) definem os ERPs como sendo sistemas que integram as áreas funcionais das empresas através de um banco de dados em comum.

Dessa maneira, os dados são guardados numa base que trabalha como uma central, armazenando, compartilhando e fazendo as informações serem disseminadas entre todos os departamentos da organização, que por sua vez passam a ter informações essenciais em tempo real (APARICIO et al., 2018). Sendo assim, as informações que anteriormente encontravam-se desintegradas e fragmentadas em diversos sistemas e setores, agora encontram-se em um único SI, através do qual, pode ser acessada e manipulada por diferentes usuários que passam a ter visões e acessos diferentes para o mesmo sistema (LAUDON et al., 2012).

Anzilago et al. (2017) ressaltam que esse tipo de sistema é utilizado pelas organizações para dar aos gestores uma visão global das operações, contribuindo de forma significativa para que ocorra a melhoria na competitividade do negócio da empresa. Para tanto, o que se busca através do uso de um sistema integrado é obter a informação correta, no momento exato e direcionada para o indivíduo certo. Tendo em vista essa integração, podem ser elencados alguns benefícios, tais como a redução de redundância de informações, a eliminação do hábito de se reinserir dados (já que ao modificar um dado no sistema, ele se reflete em todos os outros módulos) e hierarquização do acesso ao sistema, com cada funcionário tendo acesso apenas as informações que são pertinentes a sua função (FUGA et al., 2017).

Diante do exposto, pode-se observar que a adesão de uma nova ferramenta tecnológica como o ERP pode trazer ganhos significativos para uma organização, porém, para evitar impactos negativos e perdas financeiras, deve ser feita uma análise rigorosa das necessidades que se tem (avaliação das características internas) e das opções existentes no mercado (avaliação de custo-benefício e seleção de uma ferramenta que seja compatível com as características que se tem), além de cumprir adequadamente as etapas de implantação e treinamento, e de se ter um controle rigoroso aliado a manutenções periódicas e programadas (FUGA et al., 2017).

\section{METODOLOGIA}

No que se refere aos procedimentos metodológicos, o presente estudo adota a abordagem quantitativa e qualitativa. Souza et al. (2017) definem esse tipo de pesquisa como sendo uma junção entre a 
abordagem que recorre à estatística para explicação dos dados e a abordagem que lida com interpretações das realidades sociais. Essa convergência tem como objetivo atender de uma maneira melhor os universos escolhidos e fazer uma triangulação dos dados, resultando em um aumento da credibilidade e da confiabilidade do estudo.

Tratando-se da sua tipologia, o estudo é caracterizado como descritivo, expondo as principais características do sistema analisado e dos públicos utilizados como elemento de análise. Para os propósitos da pesquisa descritiva, os fatos e fenômenos devem ser extraídos do local onde realmente ocorrem/ocorreram e analisados em função das influências do ambiente, podendo ser definida em pesquisa de opinião, em estudo de caso ou em pesquisa documental.

Nesse contexto, foi escolhido como estratégia de pesquisa o estudo de caso, que segundo Yin (2015,) “investiga um fenômeno contemporâneo (o 'caso') em profundidade e em seu contexto de mundo real, especialmente quando os limites entre o fenômeno e o contexto puderem não ser claramente evidentes". Fonseca (2002) diz que um estudo de caso pode ser caracterizado como um estudo de uma entidade bem definida (como uma universidade, por exemplo) e visa conhecer o como e o porquê de uma determinada situação acontecer, procurando descobrir o que há de mais essencial e característico.

Desse mesmo modo, a entidade definida foi o Centro de Ciências Sociais Aplicadas (CCSA) da Universidade Federal de Sergipe, tendo como objeto de análise o SIG, na visão dos usuários do referido Centro: docentes e gestores. Em relação aos primeiros, o CCSA é composto por 153 docentes, dos quais 136 são efetivos e 17 são substitutos. Já os gestores, são os coordenadores/chefes dos departamentos do CCSA e o próprio Diretor desse Centro Acadêmico, sendo representados por 09 gestores departamentais mais a gestora do centro, totalizando 10 gestores, tal como ilustra o Quadro 1.

Quadro 1: Quantidade de docentes e gestores do CCSA.

\begin{tabular}{|l|l|l|l|}
\hline Departamento/Centro & \multirow{2}{*}{ No de Gestor (es) } & \multicolumn{2}{l|}{ № Docentes } \\
\cline { 2 - 4 } & & Efetivos & Substitutos \\
\hline Departamento de Administração (DAD) & 01 & 20 & 03 \\
\hline Departamento de Ciência da Informação (DCI) & 01 & 09 & 02 \\
\hline Departamento de Ciências Contábeis (DCC) & 01 & 12 & 03 \\
\hline Departamento de Direito (DDI) & 01 & 27 & 02 \\
\hline Departamento de Economia (DEE) & 01 & 22 & 04 \\
\hline Departamento de Relações Internacionais (DRI) & 01 & 09 & 01 \\
\hline Departamento de Secretariado Executivo (DSE) & 01 & 09 & 01 \\
\hline Departamento de Serviços Sociais (DSS) & 01 & 18 & 00 \\
\hline Departamento de Turismo (DTUR) & 01 & 10 & 01 \\
\hline Centro de Ciências Sociais Aplicadas (CCSA) & 01 & 136 & 17 \\
\hline
\end{tabular}

Para seleção da amostra em relação ao público docente, foi utilizada a amostragem não probabilística por acessibilidade, baseada nos indivíduos disponíveis que se dispuseram a participar da pesquisa no período de 27 de janeiro de 2020 à 10 de fevereiro de 2020. No tocante aos gestores, o estudo foi aplicado em todo o universo, Direção do Centro e os Chefes dos Departamentos do CCSA listados no Quadro 1, no mesmo período.

Como uma das técnicas de coleta de dados, empregou-se o questionário, composto por questões fechadas, abertas e intervalares baseadas na escala de Likert de 5 pontos. A construção do questionário 
ocorreu por meio da utilização do aplicativo Google Forms e sua aplicação se deu através do envio do link da página de preenchimento pelo aplicativo WhatsApp e por e-mail, objetivando os docentes do CCSA do campus de São Cristóvão.

O segundo instrumento de coleta de dados utilizado foi o roteiro de entrevista semiestruturado aplicado aos Gestores do CCSA. Segundo Pope et al. (2009), a entrevista é uma ferramenta do tipo qualitativa e possui uma estrutura flexível com questões abertas que definem a área a ser explorada. Para a aplicação dessas entrevistas, foi utilizado um roteiro de perguntas semiestruturadas, sendo que esse roteiro foi formulado com um total de treze perguntas.

No tocante aos questionários, os dados foram tabulados mediante estatística descritiva simples em valores absolutos, percentuais e médias. A respeito das entrevistas, utilizou-se do mecanismo de gravação (previamente solicitado aos entrevistados e sendo devidamente autorizado), facilitando a transcrição e o registro dos achados. Ressalta-se que, para manter o anonimato dos entrevistados, foram utilizadas letras do alfabeto de A à J, protegendo identidades e garantindo segurança a eles em relação as informações obtidas.

Além disso, com a intenção de explicitar os elementos analisados nessa pesquisa, foram definidas as seguintes categorias de análise que serão apresentadas na próxima seção: perfil dos usuários; caracterização do SIG; execução de tarefas; facilidade de uso; características das informações; problemas e possíveis melhorias.

\section{RESULTADOS E DISCUSSÃO}

No âmbito educacional, o aumento na procura e na utilização de sistemas integrados de gestão tem causado uma revolução tecnológica que, segundo Souza et al. (2015), tem afetado com maior relevância as Instituições de Ensino Superior (IES), em especial no que cerne as atividades administrativas e as práticas docentes. Pode-se afirmar que as IES têm utilizado ferramentas de Tecnologias da Informação e Comunicação (TICs) de maneira cada vez mais frequente, o que pode fomentar novos espaços e novos métodos de se ter acesso à informação.

Entre as principais ferramentas, a utilização de ERPs nas instituições educacionais surge como um destaque e o ERP escolhido como objeto de estudo dessa pesquisa é o Sistema Integrado de Gestão (SIG). O SIG foi criado pela Universidade Federal do Rio Grande do Norte (UFRN) a partir do projeto Bases de Dados Integradas, que teve como intuito construir um único banco de dados para unir as áreas acadêmica, administrativa e de recursos humanos da universidade (UFRN, 2017). Assim foi criado o SIG, com três módulos iniciais: o Sistema Integrado de Atividades Acadêmicas (SIGAA), o Sistema Integrado de Gestão e Recursos Humanos (SIGRH) e o Sistema Integrado de Patrimônio, Administração e Contratos (SIPAC).

Vale ressaltar que o resultado desse sistema foi satisfatório, tanto que a sua utilização não é exclusiva para a UFRN, diferente do que poderia ter acontecido se esse sistema fosse criado e reproduzido por uma organização da iniciativa privada, ele é compartilhado e utilizado por cerca de sessenta (60) instituições e 
órgãos da administração pública, desde universidades federais (como a Federal do Rio Grande do Norte), passando por universidades estaduais, institutos federais e até por órgãos de administração federal e estadual (UFRN, 2019), conforme Figura 1.

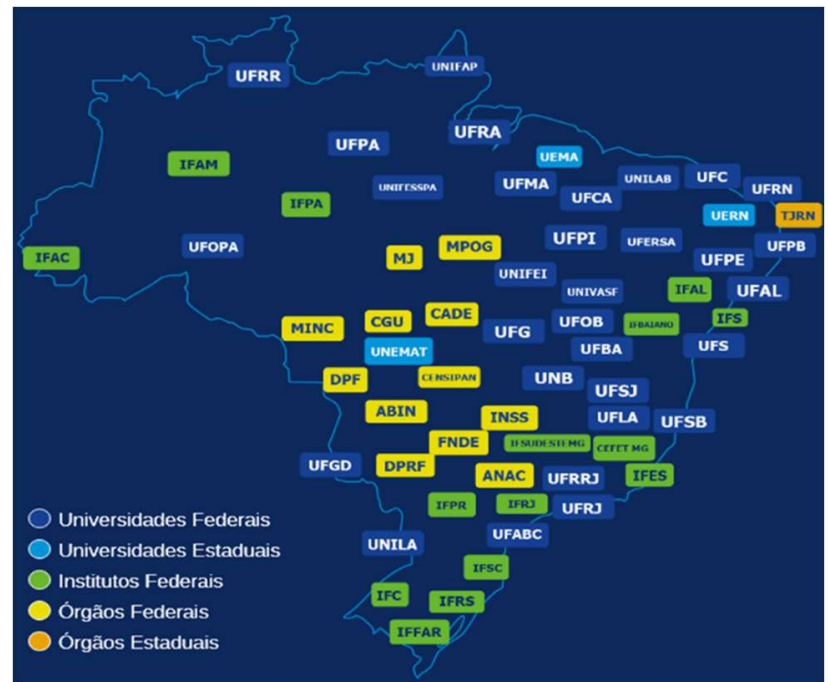

Figura 1: Mapa de utilização do SIG pelo Brasil. Fonte: Superintendência de Informática da UFRN (2019).

Diante da grande adesão desse sistema, um fato que se deve salientar, é que cada instituição possui características próprias em relação à utilização do SIG e de seus módulos, ou seja, o SIG utilizado na Universidade Federal do Rio de Janeiro (UFRJ) não necessariamente possui os mesmos módulos utilizados na Universidade Federal da Bahia (UFBA), e mesmo que sejam os mesmos módulos, provavelmente possuem características e funcionalidades diferentes. Além disso, deve-se citar que de forma geral além dos módulos que foram elencados anteriormente (SIPAC, SIGAA e SIGRH), existem diversos outros. Entre eles estão: o Sistema Integrado de Gestão da Administração e Comunicação (SIGADMIN); o Sistema Integrado de Gestão de Eleições (SIGEleição); o Sistema Integrado de Gestão de Planejamento e de Projetos (SIGPP); e o Sistema Integrado de Gestão Eletrônica de Documentos (SIGED).

Por meio do que foi exposto, ressalta-se que não serão tratados nesse estudo todos os módulos existentes do SIG, mas sim os módulos que são utilizados na Universidade Federal de Sergipe que, a partir do ano de 2009, firmou uma parceria com a Universidade Federal do Rio Grande do Norte, objetivando a execução de um projeto de implantação de sistemas informatizados de gestão (UFS, 2012). Diante da notória expansão da UFS, tornou-se imprescindível a aquisição de um sistema integrado de informações com o propósito de modernizar a gestão e agilizar os processos administrativos. Desde então, 5 módulos foram implantados nessa instituição e são eles o SIGAA, SIPAC, SIGRH, SIGADMIN e SIGEleição. O setor responsável pela manutenção e pelo acompanhamento do SIG é a Superintendência de Tecnologia da Informação (STI) da universidade, que controla, investe e apoia o uso de tecnologias de informação na instituição.

Tratando da análise dos dados quantitativos do perfil dos usuários, de um total de 153 docentes ativos no CCSA, 41 participaram de maneira voluntária, o que representa $27 \%$ do total. Desses, 90\% (38) são professores efetivos e $10 \%$ (3) substitutos, com maior representatividade do sexo masculino (56\%), com ano de ingresso na instituição entre 2010 a 2019 (85\%) e docentes do curso de Administração (29\%), ressalta-se 
Sistema Integrado de Gestão (SIG): Análise da percepção dos docentes e gestores dos cursos do Centro de Ciências Sociais Aplicadas da

que nenhum departamento ficou fora da pesquisa. No que cerne à categoria analítica relativa à caracterização do SIG, a primeira pergunta referiu-se ao recebimento ou não de treinamentos oriundos da UFS acerca de como utilizar o SIG ou pelo menos algum dos seus módulos e, nesse quesito, $63,4 \%$ dos participantes (26 docentes) informaram que não receberam nenhum tipo de treinamento.

Em relação à utilização dos módulos do SIG, os que mais obtiveram destaque foram o SIGAA, o SIGRH e o SIPAC, que já foram utilizados por $100 \%$ (41), 90\% (37) e $83 \%$ (34) dos docentes, respectivamente. No que se refere à frequência de utilização, 63\% (26 docentes) informaram utilizar o SIGAA uma ou mais vezes por dia. Em relação ao SIGRH, 56\% fazem acesso de maneira esporádica, acontecendo uma vez por mês (13 docentes) ou uma vez por semestre (10 docentes). Assim como o SIPAC, 29\% (12 docentes) uma vez por mês e 20\% (8 docentes) uma vez por semestre. Já em relação ao SIGEleição e ao SIGADMIN, a maioria dos participantes informaram nunca ter utilizado esses sistemas, com $80 \%$ e $54 \%$ (33 e 22 docentes) indicando essa opção, respectivamente.

Se tratando da execução de tarefas em cada um dos módulos do SIG, os participantes tinham a opção de citar um ou mais exemplos (então a soma total de respostas não necessariamente precisaria ser igual a 41), sendo assim, em relação ao SIGAA, as seguintes atividades tiveram maior destaque: lançamento e acompanhamento de frequência ( 25 indicações); inserção de arquivos e materiais para o acompanhamento das aulas e desenvolvimento de atividades (17 indicações); lançamento e acompanhamento de notas (15 indicações); inserção e acompanhamento do cronograma de aulas (15 indicações); e comunicação com os alunos (15 indicações). No que tange o SIPAC, as principais atividades mencionadas foram acompanhamento e envio de processos eletrônicos (25 indicações), e a análise, confecção e recebimento de memorandos (10 indicações). É importante ressaltar que parte dos respondentes informaram não desenvolver nenhuma tarefa nesse módulo do sistema (4 indicações).

Sobre o SIGRH, as atividades que se destacaram foram o acompanhamento e a solicitação de férias (22 indicações) e a atualização de dados cadastrais (7 indicações). Já em relação ao SIGEleição, apenas 6 docentes informaram que utilizam ou que já utilizaram esse módulo do sistema para votar em algum tipo de eleição. Em relação ao SIGADMIN as indicações de utilização também foram baixas, 4 docentes indicaram que o utilizam para se comunicar de maneira geral com outros setores da instituição. Tal fato ocorre em função do docente já ter ocupado cargo de Gestão.

Ao tratar da facilidade de uso do SIG, conforme Quadro 2, destaca-se a predominância de respostas nas escalas fácil e regular, mostrando no geral a facilidade de uso do sistema nos itens analisados. No entanto, merece destaque ainda a presença de dificuldade relatada para upload no sistema (8 respondentes consideraram um grau de dificuldade).

No tocante as características das informações, fundamentadas por Stair et al. (2011), questionou-se a respeito da velocidade, segurança, confiança e flexibilidade das informações geradas pelo sistema. Assim, pôde-se constatar que o SIG foi avaliado por 39\% (16 docentes) como rápido e 37\% (15 docentes) como regular na velocidade de processamento. Indo além, mais de $75 \%$ dos respondentes (31 docentes) 
apontaram que se sentem seguros em receber e/ou enviar informações através do sistema, assim como, 78\% (32 docentes), demonstraram confiança nas informações extraídas do SIG, seguido de 63\% (26 docentes) que apontaram que as informações são acessíveis, corroborando o que consta na literatura à cerca de qualidade da informação. Por fim, no tocante a flexibilidade, foi apontado uma variância nas repostas: 37\% (15 docentes) afirmaram que era regular, 34\% (14 docentes) afirmaram que o sistema era flexível e 29 \% (12 docentes) indicaram que o SIG não é flexível, ou seja, segundo a visão deles, o sistema padroniza os relatórios gerados.

Quadro 2: Avaliação da facilidade de uso do SIG.

\begin{tabular}{|l|l|l|l|l|l|l|l|}
\hline & Muito fácil (1) & Fácil (2) & Regular (3) & Difícil (4) & Muito difícil (5) & Média & Total \\
\hline Primeiro acesso & 4 & 17 & 13 & 5 & 2 & 2,61 & 41 \\
\hline Funcionalidades do SIG & 6 & 23 & 8 & 2 & 2 & 2,10 & 41 \\
\hline Intuitividade & 2 & 22 & 12 & 4 & 1 & 2,51 & 41 \\
\hline Upload de arquivos & 9 & 13 & 11 & 4 & 4 & 2,54 & 41 \\
\hline Download de arquivos & 11 & 16 & 8 & 4 & 2 & 2,27 & 41 \\
\hline Geração de relatórios & 11 & 19 & 6 & 5 & 0 & 2,12 & 41 \\
\hline
\end{tabular}

Tratando da percepção dos docentes em relação a existência de problemas e possíveis melhorias. Destaca-se que 68,3\% (28 docentes) informaram ter problemas com acesso ao sistema devido a manutenções do sistema em horário de expediente ou manutenções não programadas (ou, se programadas, que pelo menos não foram sinalizadas para os usuários) e lentidão do sistema.

Já sobre as possíveis melhorias, foi solicitado aos professores que indicassem se na concepção deles havia algum ponto que deveria ser modificado ou melhorado no SIG, obtendo 90,2\% (37 docentes) de concordância. Desse percentual, por ser questão aberta, os registros que mais se destacaram foram: alteração do layout do sistema com o intuito de fazer o SIG ser mais acessível e atrativo, melhorando a interface gráfica; aumento da velocidade do sistema; simplificação de processos; e integração do SIG com outros sites e sistemas. Inclusive, no que se refere a esse último tópico, um dos relatos surpreendeu, pois, um dos docentes afirmou que há falta de integração entre os próprios módulos do SIG o que faz com que os usuários tenham de inserir uma mesma informação mais de uma vez.

Partindo para a análise dos dados das entrevistas com os gestores dos departamentos vinculados ao CCSA e a própria direção do centro, em relação ao perfil dos usuários aferiu-se que a maior parte dos gestores apresentou idades entre 39 e 46 anos, porém com uma média geral de 48 anos devido as idades extremas apresentadas (o gestor mais novo declarou possuir 39 anos e o mais velho 70 anos). Tratando sobre o nível de escolaridade, o que se pôde aferir é que todos são mestres ou doutores, sendo que a maior parte (7 dos 10 participantes) informou possuir o título de doutor. Outra informação extraída dos participantes se refere ao tempo de serviço no cargo atual, e com exceção de 4 gestores, pôde-se verificar que a maioria se encontrava no seu primeiro mandato (o mandato de gestor normalmente dura 2 anos podendo ser renovado por mais 2 anos). De maneira complementar, 6 dos 10 gestores indicaram não terem ocupado nenhum outro cargo de caráter administrativo na universidade. Essas informações sugerem que mesmo com um elevado grau de instrução, entre os participantes há pouca experiência no que se diz respeito a ocupar cargos de gestão. 
Já em relação a caracterização do SIG, mais especificamente sobre os treinamentos acerca de como utilizar o SIG, encontrou-se os seguintes relatos:

Não, existe um manual, mas a gente aprende mesmo com a prática. Eu, por exemplo, só comecei a ter uma certa facilidade com o sistema depois de uns 4 ou 5 meses utilizando. (Gestor F, 2020)

[...] como gestor eu aprendi boa parte do que sei com a antiga gestora, como em uma fase de transição, que foi muito interessante. Essa iniciativa partiu da antiga gestora. (Gestor J, 2020)

Mesmo entre aqueles que informaram terem tido algum tipo de treinamento proveniente da UFS, alguns fizeram observações sobre a necessidade de se haver uma capacitação mais adequada:

[...] houveram treinamentos sim, mas todos foram excessivamente triviais e não acrescentaram nada no aprendizado. As questões mais complexas acabavam não sendo explicadas e aí você tem que aprender por tentativa e erro. A partir daí eu parei de ir nesses treinamentos. (Gestor G, 2020)

[...] de maneira geral, acho que esse é um dos grandes pontos a serem considerados, pois quando a gente entra na universidade, é jogado para trabalhar, então nem quando eu entrei como docente, nem quando eu entrei como vice chefe, nem quando eu entrei como chefe eu tive algum tipo de treinamento efetivo. Então eu acredito que muitas vezes a gente erra ou a gente subutiliza o sistema por conta disso. (Gestor B, 2020)

Sobre a utilização dos módulos do sistema, por tais gestores atuarem também como docente, o módulo SIGAA teve destaque. Entretanto, ressaltaram que na função ocupada fazem uso rotineiro do módulo SIPAC e mensalmente do SIGRH. Merece destaque a não usabilidade do módulo SIGADMIN, dos 10 entrevistados, metade relatou que usa esporadicamente.

Na categoria analítica relativa à execução de tarefas, os gestores foram indagados sobre as principais tarefas que desenvolvem em cada um dos módulos do SIG e assim como aconteceu na aplicação dos questionários, os chefes de departamento tinham a opção de citar um ou mais exemplos, ou ainda, não citar exemplos. Em relação ao SIGAA as principais atividades apontadas foram: criação, solicitação e acompanhamento das turmas (5 indicações); acompanhamento e homologação de estágios (4 indicações); e a validação de projetos de extensão ( 2 indicações). No que tange o SIPAC, as principais atividades mencionadas foram: análise, acompanhamento, resposta e envio de processos diversos (5 indicações); e validação, envio e recebimento de memorandos (8 indicações).

As principais atividades que foram apontadas como as desenvolvidas no SIGRH foram: acompanhamento e lançamento de férias dos professores e servidores (10 indicações); lançamento e acompanhamento da frequência dos servidores e docentes do departamento (6 indicações); e o acompanhamento dos contratos dos professores substitutos (2 indicações). Em relação ao SIGEleição, foi indicada apenas uma atividade como sendo realizada, e foi a de votar em eleições (3 indicações). Já em relação ao SIGADMIN dos 5 gestores que informaram utilizar ou já ter utilizado esse sistema em algum momento, apenas 3 se pronunciaram, e citaram duas atividades principais: a ação de se comunicar com outros departamentos e funcionários da instituição ( 2 indicações); e liberar funções administrativas para os servidores do departamento ( 2 indicações).

As respostas acima apresentadas reiteram que dos cinco módulos existentes, 3 possuem maior 
notoriedade entre os entrevistados (SIGAA, SIPAC e SIGRH), e assim como exposto nos casos dos docentes isso ocorre pela associação existente entre as suas características e as necessidades da função dos participantes.

Questionados sobre a facilidade de uso e importância do SIG, merecem destaque as seguintes falas:

Eu acho que o SIG é de suma importância, ele é imprescindível, e em qualquer lugar que você esteja, você consegue acessar os módulos do SIG e consegue trabalhar. (Gestor A, 2020)

Os módulos do SIG são de suma importância, inclusive, sem eles, acredito que não daria para trabalhar hoje em dia, porque existe toda uma estruturação em volta do SIG que faz da universidade dependente. (Gestor D, 2020)

Um dos gestores ainda complementa essas falas afirmando que o SIG assumiu um papel de direcionar a universidade para caminhos futuros: "É um norte, um farol” (Gestor E, 2020). O discurso apresentado pelo Gestor E, e também pelos demais gestores, indica que o sistema conduz a universidade para uma evolução processual constante e que vai ao encontro do desenvolvimento da TI de uma maneira global. Seguindo essa mesma linha de pensamento, o Gestor I apontou que uma das vantagens do SIG se encontra na dinamicidade que é dada para os processos da UFS: "O SIG torna mais célere as comunicações entre os departamentos" (Gestor I, 2020).

Ao tratar das características das informações objetivou-se auferir a percepção dos gestores em relação à segurança, confiabilidade, acessibilidade, confiabilidade e flexibilidade. Destaca-se os seguintes relatos que corroboram a parte descritiva da percepção dos professores de que a informação é segura e confiável:

A universidade avisa que não solicita esse tipo de dado e informa que se isso for feito é porque não são eles. Sendo assim, há essa preocupação deles, e eu me sinto seguro em relação aos trabalhos que são feitos dentro do sistema. (Gestor H, 2020)

Em função do que é público ou não, no próprio sistema existem itens que nos dão opções para que a comunicação, ou o documento, sejam ostensivos ou sigilosos". (Gestor I, 2020)

No tocante à acessibilidade, os gestores $\mathrm{E}$ e $\mathrm{F}$ afirmaram que quando se trata de tarefas cotidianas $\mathrm{e}$ simples as informações são de fácil acesso, porém, quando são tarefas não rotineiras, surgem dificuldades relacionadas à acessibilidade: “As coisas mais importantes são relativamente fáceis de se conseguir, mas tem coisas complicadas" (Gestor F, 2020).

No quesito relativo a flexibilidade, somente dois gestores não se posicionaram, sendo predominante a concepção que o sistema é engessado. Isso pode ser verificado através das falas dos Gestores B, D e G, respectivamente: "não, pois há uma certa dificuldade em gerar determinados relatórios mais detalhados. Não dá para fugir muito dos padrões pré-estabelecidos, realmente há um engessamento" (Gestor B, 2020); “o sistema é um pouco engessado, tem situações que você tenta configurar um relatório especial, você quebra a sua cabeça e não consegue" (Gestor D, 2020); "os relatórios são muito estereotipados, você não consegue gerar relatórios específicos e isso é um problema" (Gestor G, 2020).

De maneira complementar, o Gestor A exemplificou como acontece essa inflexibilidade do sistema:

Agora por exemplo, eu estou tendo que fazer um inventário, e eu tenho como retirar um relatório por número de tombamento ou por nome. Se for necessário por alguma outra 
referência, eu não consigo. Em outras situações, eu acabo tendo que enviar mensagens para os alunos ou docentes de algum determinado departamento, e eu não consigo fazer isso por curso, daí eu tenho que enviar para todos os discentes ou docentes do centro. (Gestor A, 2020)

No tocante aos problemas e possíveis melhorias no sistema, 8 dos 10 gestores afirmaram ver problemas ou limitações no SIG, tal como narrou o Gestor G: “[...] excesso de digitalização das coisas, lentidão e muitos erros no sistema" (Gestor G, 2020). Seguindo essa mesma linha de pensamento, o Gestor H apresentou certa insatisfação no quesito velocidade: "Eu sinto dificuldade no que se refere a velocidade do sistema, para gerar, baixar ou inserir arquivos no sistema. Além disso, alguns relatórios como o de histórico, são muito demorados" (Gestor H, 2020).

Outros participantes concordaram em ver limitações no sistema, porém, suavizaram essas questões afirmando que veem de uma maneira geral, mais benefícios do que malefícios. É o caso dos gestores $A$ e $E$, respectivamente: "Sim, eu vejo, mas não são coisas prejudiciais e sim limitações" (Gestor A, 2020); "Sim, eu enxergo, mas também reconheço que ele nos ajuda e tem evoluído e isso faz parte do processo. Isso é normal" (Gestor E, 2020).

Por fim, o Gestor B trouxe mais uma vez questões relativas à estrutura organizacional da universidade: "[...] tem algumas coisas, pequenos detalhes de algumas funcionalidades que dariam para ser facilitadas, mas esbarramos em um certo engessamento do sistema, que não depende nem da STI, mas sim da autorização da Reitoria e da Pró-Reitoria" (Gestor B, 2020). No entanto, esse mesmo gestor apontou sugestões e possíveis melhorias, principalmente sobre o quesito manutenção:

Em relação as manutenções, elas ocorrem meio que de surpresa e durante o expediente. Mas o que a gente tem que pensar é que não é passado o motivo da manutenção, se for uma manutenção preventiva, que normalmente já se sabe com antecedência que deve ser feita, daria para fazer em um horário diferenciado, porém a gente sabe que com tecnologia da informação muitas vezes acontecem problemas com hardware ou software que tem que ser resolvido na hora, senão fica pior, e aí tem que ser resolvido na hora. (Gestor B, 2020).

Sendo assim, como já aferido em relatos dos questionários docentes, pôde-se verificar que manutenções não programadas, ou não avisadas, criam um verdadeiro transtorno para a comunidade acadêmica. Além disso, o Gestor $D$ indicou que na sua visão, a melhor solução em relação aos problemas existentes no SIG se resume em: "[...] capacitar as pessoas para que elas tenham mais conhecimento sobre o sistema o que faria surgir mais possibilidades e eliminar os problemas" (Gestor D, 2020). Além de falas que reafirmaram uma necessidade maior no que tange as questões da velocidade do sistema: "[...] o sistema precisa ser mais ágil em relação as suas informações" (Gestor H, 2020).

\section{CONCLUSÕES}

Essa pesquisa teve por objetivo analisar o Sistema Integrado de Gestão da Universidade Federal de Sergipe no tocante a execução de tarefas e tomada de decisão, na percepção dos docentes e gestores dos cursos do Centro de Ciências Sociais Aplicadas. Conclui-se a respeito da importância do SIG para a comunidade acadêmica em que a maioria dos gestores e dos docentes informou que essa ferramenta auxilia de maneira positiva no desempenho das suas atividades e nos trâmites de informações da universidade como 
um todo. Essas afirmações indicam que há uma significativa relação entre os usuários e o sistema mediante várias vantagens, tal como a celeridade na troca de informação.

Ademais, pode-se afirmar que o SIG da UFS é um sistema que possui um grau relevante de qualidade, e que tem um bom nível de aceitação por parte dos respondentes no tocante a segurança, acessibilidade e confiabilidade. Todavia, tratando-se dos aspectos negativos, o mesmo foi caracterizado como um sistema abaixo do esperado no que se refere a velocidade e flexibilidade, principalmente na geração dos relatórios. Indo além, outros três pontos merecem destaque como problemas relacionados ao sistema. $\mathrm{O}$ primeiro refere-se as manutenções não programadas do sistema que foram salientadas pelos dois públicos participantes da pesquisa. Tendo em vista essas situações recorrentes, deve-se salientar que, segundo Ferro et al. (2013), se não forem muito bem programadas e ajustadas, paradas para manutenções, modificações ou atualizações do sistema, podem trazer prejuízos para a organização.

O segundo refere-se à capacitação, pôde-se perceber que ao ingressar na Universidade Federal de Sergipe, grande parte dos participantes dessa pesquisa (de ambos os públicos), não receberam nenhum tipo de treinamento. Mesmo dentre os que receberam, houveram críticas relativas à qualidade dessas capacitações, indicando que a não utilização ou a subutilização do sistema acontece por conta da baixa qualidade ou falta de treinamento.

O terceiro diz respeito à integração do SIG com outros sites e entre os seus próprios módulos. Em um dos relatos docentes foi afirmado que, em determinados momentos, os usuários necessitam inserir a mesma informação mais de uma vez em módulos diferentes, o que mostra uma falha no sistema, visto que, na realidade o intuito de ERPs, como o SIG, é integrar e não gerar retrabalho.

Assim, percebe-se que o objetivo da pesquisa foi atendido, sendo possível analisar o SIG no tocante a execução de tarefas e tomada de decisão, na percepção dos docentes e gestores dos cursos do Centro de Ciências Sociais Aplicadas. Os dados apontam a utilização na combinação desses dois perfis e as características do sistema, podendo ser fatores observáveis na organização.

Dentre as principais limitações apresentadas nessa pesquisa, destaca-se o tamanho da amostra dos docentes, uma vez que não foi possível fazer a coleta com todos os professores do Centro Acadêmico estudado. Considera-se que o número de participantes foi o mínimo necessário para realizar a pesquisa, e que se a adesão tivesse sido maior, os resultados poderiam ser ainda mais contundentes, de maneira semelhante caso fosse ampliado para outros centros. Desse modo, como dito por um dos gestores participantes desse estudo, "[...] para que o SIG possa atender ao maior número possível de realidades, o ideal seria haver um diálogo maior entre todas as partes" (Gestor E, 2020).

Para que isso ocorra, é imprescindível que sejam realizados mais estudos como esse, e que se levem os resultados obtidos aos setores competentes, pois eles possuem o poder para pleitear alterações que possam beneficiar toda a comunidade acadêmica da Instituição. Tendo isso em vista, a pesquisa pode ser continuada e ampliada em várias frentes, portanto, além da ampliação do universo estudado, recomenda-se envolver em pesquisas futuras o setor responsável pela administração do SIG, a Superintendência de 
Tecnologia da Informação, além de buscar o entendimento de como as questões discutidas neste estudo são gerenciadas em outras organizações que vivenciam ambientes acadêmicos públicos ou privados.

\section{REFERÊNCIAS}

ANDRADE, D. G.; FALK, J. A.. Eficácia de sistemas de informação e percepção de mudança organizacional. Revista de Administração Contemporânea, Curitiba, v.5, n.3, p.5384, 2001.

ANZILAGO, M.; ZANIN, D. F.; BEZERRA, C. A.. Vantagem competitiva na utilização de sistemas de informação: Enterprise Resource Planning - ERP. In: CONGRESSO DA ASSOCIAÇÃO NACIONAL DE PROGRAMAS DE PÓSGRADUAÇÃO EM CIÊNCIAS CONTÁBEIS, 11. Anais. Belo Horizonte: ANPCONT, 2017. p.636-650.

APARICIO, M.; RAPOSO, J.; COSTA, C. J.. A utilização de ERP em contexto de Ensino Superior. In: IBERIAN CONFERENCE ON INFORMATION SYSTEMS AND TECHNOLOGIES, 13. Anais. Lisboa: ISCTE-IU, 2018.

FERNÁNDEZ, M. V.; GOMES, L. I. E.; MARQUES, M. B. Perspectiva teórica e metodológica em sistemas de informação complexos. Páginas A\&B: Arquivos e Bibliotecas, Lisboa, n.4, p.3-21, 2015.

FERRO, D. A.; FERREIRA NETO, M. A importância do sistema integrado de gestão empresarial para as instituições privadas ou públicas. Monografia (Bacharelado em Administração) - Pontifícia Universidade Católica de Goiás, Goiânia, 2013.

FONSECA, J. J. S.. Metodologia da pesquisa científica. Fortaleza: UEC, 2002.

FUGA, M. A.; FARIAS, F.; TREGNANSIN, R.; OLEA, P. M.. Análise da utilização de sistemas de informação Enterprise Resource Planning (ERPs) em instituições de ensino. In: MOSTRA DE INICIAÇÃO CIENTÍFICA, PÓS-GRADUAÇÃO, PESQUISA E EXTENSÃO - PROGRAMA DE PÓS-GRADUAÇÃO EM ADMINISTRAÇÃO, 17. Anais. Caxias do Sul: UCS, 2017.

GORLA, N.; SOMERS, T. M.; WONG, B.. Organizational impact of system quality, information quality, and service quality. Journal of Strategic Information Systems, v.19, p.207-228, 2010.

JANNUZZI, C. A. S. C.; FALSARELLA, O. M.; SUGAHARA, C. R.. Sistema de informação: um entendimento conceitual para a sua aplicação nas organizações empresariais. Perspectivas em Ciência da Informação, v.19, n.4, p.94-117, 2014.

KROENKE, D. M.. Sistemas de informação gerenciais. São Paulo: Saraiva, 2017.

LAUDON, K. C.; LAUDON, J. P.. Sistemas de informação gerenciais. 9 ed. São Paulo: Pearson, 2012.
O'BRIEN, J. A.. Sistemas de informação e as decisões gerenciais na era da internet. 3 ed. São Paulo: Saraiva, 2011.

POPE, C.; MAYS, N.. Pesquisa qualitativa na atenção à saúde. 3 ed. Porto Alegre: Artmed, 2009.

ROCHA NETO, A. F.; LIMA, G. A. F.. Turma virtual do SIGAA como ferramenta de apoio ao ensino. Natal: UFRN, 2009.

SOUZA, K. K.; KERBAUY, M. T. M.. Abordagem quantiqualitativa: superação da dicotomia quantitativa-qualitativa na pesquisa em educação. Educação e Filosofia, Uberlândia, v.31, n.61, p.21-44, 2017

SOUZA, M. N. A.; MONTEIRO, A. J.. Os docentes da Universidade Federal do Ceará e a utilização de alguns dos recursos do Sistema Integrado de Gestão de Atividades Acadêmicas (SIGAA). Ensaio: Avaliação e Políticas Públicas Educacionais, Rio de Janeiro, v.23, n.88, p.611-630, 2015.

STAIR, R. M.. Princípios de sistemas de informação: uma abordagem gerencial. 8 ed. Rio de Janeiro: Cengage Learning, 2001.

STAIR, R. M.; REYNOLDS, G. W.. Princípios de sistemas de informação. 9 ed. São Paulo: Cengage Learning, 2011.

TURBAN, E.; RAINER JUNIOR, R. K.; POTTER, R. E.. Introdução a sistemas de informação: uma abordagem gerencial. Rio de Janeiro: Elsevier, 2007.

UFS. Universidade Federal de Sergipe. Desenvolvido pela ASCOM-UFS. UFS lança o Sipac, mais um módulo do sistema integrado. São Cristóvão: UFS, 2012.

UFRN. Universidade Federal do Rio Grande do Norte. Desenvolvido pela Superintendência de Informática da UFRN. Cooperações Técnicas. Natal: UFRN, 2017.

UFRN. Universidade Federal do Rio Grande do Norte. Desenvolvido pela Superintendência de Informática da UFRN. Sistemas Institucionais Integrados de Gestão - SIG. Natal: UFRN, 2019

YIN, R. K.. Estudo de caso: planejamento e métodos. 5 ed. Porto Alegre: Bookman, 2015.

ZANUZ, F. M. B.. Impacto da qualidade da informação no trabalho individual: análise do uso de um sistema de informação na administração pública. Dissertação (Mestrado) - Universidade Federal do Paraná, Curitiba, 2015.

ZIULKOSKI, L. C. C.. Integração do moodle com o banco de dados institucional da UFRS. Porto Alegre: UFRS, 2010.

A CBPC - Companhia Brasileira de Produção Científica (CNPJ: 11.221.422/0001-03) detém os direitos materiais desta publicação. Os direitos referem-se à publicação do trabalho em qualquer parte do mundo, incluindo os direitos às renovações, expansões e disseminações da contribuição, bem como outros direitos subsidiários. Todos os trabalhos publicados eletronicamente poderão posteriormente ser publicados em coletâneas impressas sob coordenação da Cognitionis Publishing, da Companhia Brasileira de Produção Científica e seus parceiros autorizados. Os (as) autores (as) preservam os direitos autorais, mas não têm permissão para a publicação da contribuição em outro meio, impresso ou digital, em português ou em tradução. 